\title{
Does low angiopoietin-1 predict adverse outcome in sepsis?
}

\author{
Sascha David', Matijs van Meurs',2 and Philipp Kümpers* \\ See related research by Mankhambo et al., http://ccforum.com/content/14/3/R91
}

\begin{abstract}
Endothelial injury has emerged as a crucial early event in the pathogenesis of microcirculatory dysfunction, capillary leakage and multiorgan dysfunction syndrome. The endothelial-specific angiopoietin (Ang)/Tie2 ligand-receptor system has been identified recently as a nonredundant regulator of endothelial responsiveness. Ang- 1 is a Tie 2 agonist and promotes endothelial stabilization and quiescence, whereas Ang-2 is a Tie2 antagonist and promotes endothelial activation, destabilization, and inflammation. While the mediator function of both Ang-1 and Ang-2 has been well established in preclinical research, only Ang-2 has been identified as a clinically useful biomarker in the critical care arena. In the previous issue of Critical Care, Mankhambo and colleagues report on angiogenic factors in Malawian children with severe bacterial infection. Among those children, diminished levels of the vessel-protective factor Ang-1 remained a significant predictor of outcome after multivariate adjustment. Whether low Ang-1 represents an important risk factor of adverse outcome in critically ill adults remains to be seen.
\end{abstract}

Not until the nineteenth century was it generally agreed that arteries and veins are connected by capillaries and that these capillaries indeed have a cellular wall, the socalled endothelium. At this point in time, one was convinced that the endothelium was a passive inner layer of blood vessels, not participating in any kind of active processes. Nowadays, better understanding of physiological processes involving the endothelium (for example, coagulation, hemostasis, and so forth) has led to

*Correspondence: pkuempers@gmx.de

${ }^{3}$ Department of Medicine D, Division of General Internal Medicine, Nephrology, and Rheumatology, University Hospital Münster, Albert-Schweitzer-Strasse 33, 48149 Münster, Germany

Full list of author information is available at the end of the article appreciation of the endothelium as in independent and active organ system. During the past decade, endothelial injury has attracted much attention - being a crucial event in the pathogenesis of experimental septic organ dysfunction. As a consequence, several circulating factors released by the activated endothelial cells, neighboring support cells (such as vascular smooth muscle cells and pericytes), or platelets have been identified as biomarkers of outcome in sepsis.

In the previous issue of Critical Care, Mankhambo and colleagues report on circulating levels of vascular endothelial growth factor (VEGF), platelet-derived growth factor, fibroblast growth factor, angiopoietin (Ang)-1 and Ang-2 in Malawian children with severe infection [1]. They found robust increases in VEGF, platelet-derived growth factor, fibroblast growth factor and Ang-2 levels (twofold to ninefold), whereas Ang-1 was modestly decreased by only $15 \%$ (not significant). Survivors had lower Ang-2 and higher platelet-derived growth factor and Ang-1 levels, respectively (Figure 1). Only (low) Ang-1, however, was significantly associated with mortality after multivariate adjustment.

In 1996 the Ang/Tie2 ligand-receptor system was discovered as the second class of vascular-specific receptor tyrosine kinases (the first being the VEGF/VEGFreceptor system). Classically thought of as an important regulator in vessel maturation and remodeling, recent studies demonstrated that the Ang/Tie2 system not only regulates angiogenesis but also controls endothelial inflammation in a nonredundant manner [2]. Ang-1 and Ang-2 are antagonistic ligands that bind to the extracellular domain of the Tie2 receptor, which is almost exclusively expressed by endothelial cells. Binding of the agonist Ang-1 maintains vessel integrity, inhibits vascular leakage, suppresses inflammatory gene expression, and prevents recruitment and transmigration of leukocytes [3]. In contrast, binding of Ang-2 to Tie2 disrupts protective Ang-1 signaling and facilitates endothelial inflammation, most probably via preventing Ang-1 from phosphorylating Tie2 [4]. Ang-2 was identified recently as a strong and independent predictor of outcome in early and later stages of critical illness in adults [5-9]. 


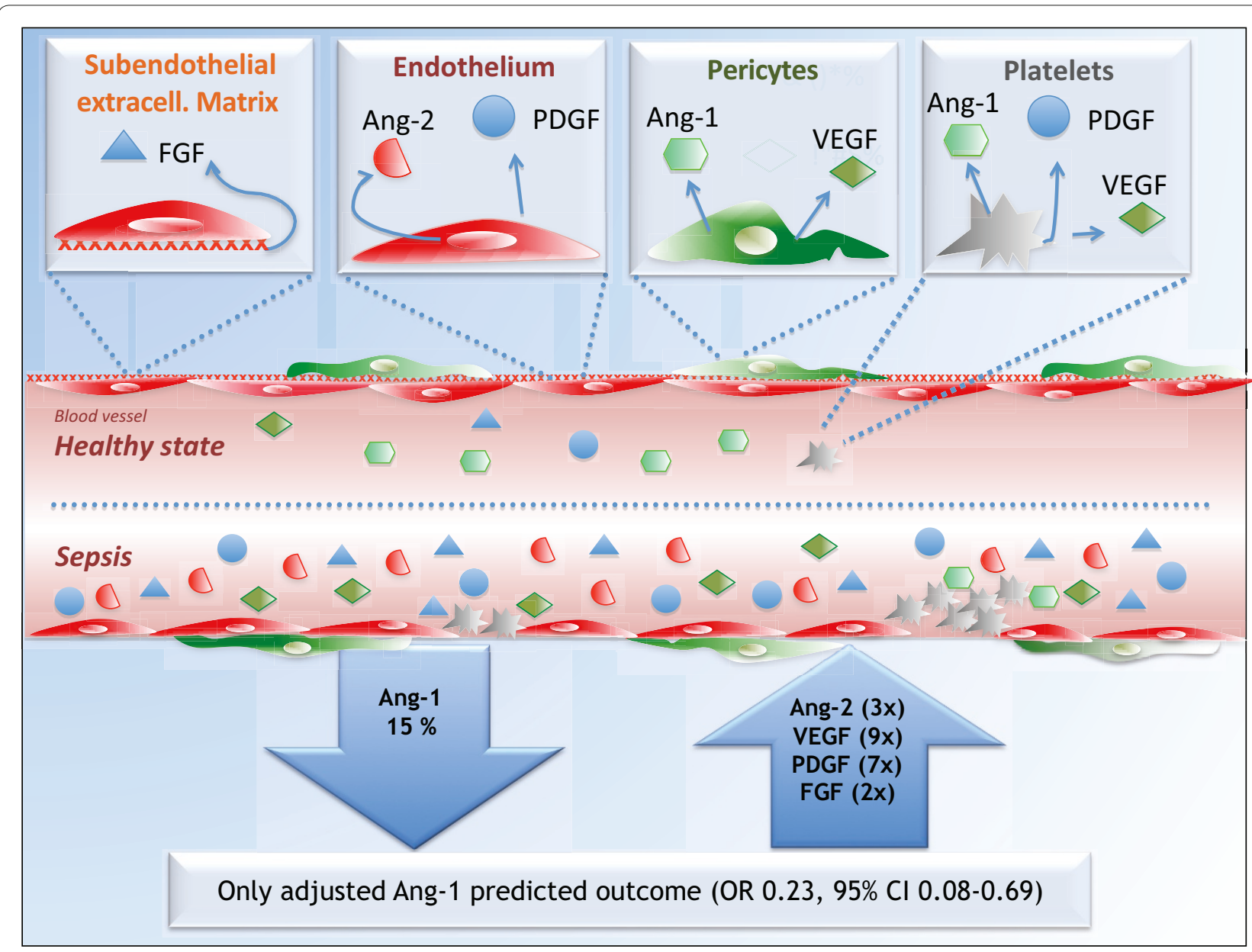

Figure 1. Schematic model of circulating levels of angiogenic factors in Malawian children with severe infection. Schematic model of the main findings for circulating levels of vascular endothelial growth factor (VEGF), platelet-derived growth factor (PDGF), fibroblast growth factor (FGF), angiopoietin (Ang)-1 and Ang-2 in Malawian children with severe infection from the study by Mankhambo and colleagues [1]. Cl, confidence interval; OR, odds ratio.

Clinical data from children with severe infection or sepsis are still very limited. Giuliano and coworkers were the first to report that Ang-2 levels were significantly elevated and Ang-1 levels were significantly decreased in children with septic shock compared to children with either systemic inflammatory response syndrome or sepsis [10]. Only circulating Ang-2, however, correlated with disease severity and outcome. The same investigators analyzed Ang-2 release in children after cardiopulmonary bypass surgery [11]. They identified a close correlation of Ang-2 with surrogate markers of capillary leakage and found a relevant role of Ang-2 in predicting the intensive care unit length of stay. In contrast to septic children of the aforementioned study, circulating Ang-1 levels were normal or even elevated after cardiopulmonary bypass. Finally, recent data coming from Ugandan children indicate that Ang-2 might serve as a biomarker to discriminate uncomplicated malaria from cerebral malaria [12].

While the mediator function of both Ang-1 and Ang-2 has been well established in preclinical research, only Ang-2 has been established as a clinically useful biomarker in the critical care arena [13]. Speculation is tempting, however, on the disturbances in the Ang/Tie2 system during sepsis being a shift from the more protective Ang-1 to the more vascular disruptive Ang-2 [13]. While some authors found elevated Ang-1 levels, others detected low Ang-1 concentrations in intensive care unit patients. This finding might be explained by differences in the samples used for analysis. Unlike Ang-2, which is exclusively stored in endothelial cells, Ang-1 is not exclusively expressed by the vascular wall (Figure 1) - a high amount of Ang-1 (and VEGF) is also found in platelets [14]. False positive Ang-1 levels can 
therefore result from the ex vivo activation of platelets within serum tubes, a problem recognized only recently.

The article by Mankhambo and colleagues adds knowledge to our current understanding of Ang-1 in critical illness. Plasma Ang-1 levels were not different between patients and controls, but low Ang-1 was independently associated with poor prognosis. Therefore it is reasonable to assume that a very small decrease in circulating Ang-1 has a huge impact on outcome, and that low Ang-1 is probably not associated with mortality in a linear fashion. One might speculate that low Ang-1 per se represents an important risk factor for adverse outcome in children with sepsis. Unfortunately, the authors neither investigated the correlation between plasma Ang-1 and clinicopathologic features of multiorgan dysfunction syndrome, nor reported the sensitivity and specificity of specific Ang-1 cut-off values in predicting outcome.

With regard to the value of circulating Ang-1 as a biomarker in sepsis, several questions remain open. What is the role of Ang-1 in platelets and does plateletderived Ang-1 correlate with outcome? Is it possible to quantify Ang-1 in a compartment-specific fashion (that is, true circulating Ang-1 vs. platelet-derived Ang-1)? Is there a specific all-or-nothing cut-off value for Ang-1, or is the ratio between Ang-1 and Ang-2 critical for outcome prediction? All of these questions remain an active focus in several laboratories, including our own.

Abbreviations

Ang, angiopoietin; VEGF, vascular endothelial growth factor.

Acknowledgements

SD is a scholar of the Deutsche Forschungsgemeinschaft (DA 1209/1-1).

Competing interests

The authors declare that they have no competing interests.

\section{Author details}

${ }^{1}$ Center of Vascular Biology Research, Beth Israel Deaconess Medical Center and Harvard Medical School, 99 Brookline Avenue, Boston, MA 02215, USA. ${ }^{2}$ Department of Critical Care, University Medical Center Groningen, Hanzeplein 1, 9713 GZ Groningen, The Netherlands. 'Department of Medicine D, Division of General Internal Medicine, Nephrology, and Rheumatology, University Hospital Münster, Albert-Schweitzer-Strasse 33, 48149 Münster, Germany.

Published: 20 July 2010
References

1. Mankhambo LA, Banda DL, The IPD Study Group, Jeffers G, White SA, Balmer P, Nkhoma S, Phiri H, Molyneux EM, Hart CA, Molyneux ME, Heyderman RS, Carrol ED: The role of angiogenic factors in predicting clinical outcome in severe bacterial infection in Malawian children. Crit Care 2010, 14:R91.

2. Fiedler U, Reiss Y, Scharpfenecker M, Grunow V, Koidl S, Thurston G, Gale NW, Witzenrath M, Rosseau S, Suttorp N, Sobke A, Herrmann M, Preissner KT, Vajkoczy P, Augustin HG: Angiopoietin-2 sensitizes endothelial cells to TNF- $a$ and has a crucial role in the induction of inflammation. Nat Med 2006, 12:235-239.

3. Witzenbichler B, Westermann D, Knueppel S, Schultheiss HP, Tschope C: Protective role of angiopoietin-1 in endotoxic shock. Circulation 2005, 111:97-105.

4. Parikh SM, Mammoto T, Schultz A, Yuan HT, Christiani D, Karumanchi SA, Sukhatme VP: Excess circulating angiopoietin-2 may contribute to pulmonary vascular leak in sepsis in humans. PLoS Med 2006, 3:e46.

5. Kumpers P, Lukasz A, David S, Horn R, Hafer C, Faulhaber-Walter R, Fliser D, Haller $\mathrm{H}$, Kielstein JT: Excess circulating angiopoietin-2 is a strong predictor of mortality in critically ill medical patients. Crit Care 2008, 12:R147.

6. Kumpers P, Hafer C, David S, Hecker H, Lukasz A, Fliser D, Haller H, Kielstein JT, Faulhaber-Walter R: Angiopoietin-2 in patients requiring renal replacement therapy in the ICU: relation to acute kidney injury, multiple organ dysfunction syndrome and outcome. Intensive Care Med 2010, 36:462-470.

7. Siner JM, Bhandari V, Engle KM, Elias JA, Siegel MD: Elevated serum angiopoietin 2 levels are associated with increased mortality in sepsis. Shock 2009, 31:348-353.

8. van der Heijden M, Pickkers P, Nieuw Amerongen GP, van Hinsbergh VW, Bouw MP, van der Hoeven JG, Groeneveld AB: Circulating angiopoietin-2 levels in the course of septic shock: relation with fluid balance, pulmonary dysfunction and mortality. Intensive Care Med 2009, 35:1567-1574.

9. Kumpers P, van Meurs M, David S, Molema G, Biizet J, Lukasz A, Biertz F, Halle $H$, Zijlstra JG: Time course of angiopoietin-2 release during experimental human endotoxemia and sepsis. Crit Care 2009, 13:R64.

10. Giuliano JS, Jr, Lahni PM, Harmon K, Wong HR, Doughty LA, Carcillo JA, Zingarelli B, Sukhatme VP, Parikh SM, Wheeler DS: Admission angiopoietin levels in children with septic shock. Shock 2007, 28:650-654.

11. Giuliano JS, Jr, Lahni PM, Bigham MT, Manning PB, Nelson DP, Wong HR, Wheeler DS: Plasma angiopoietin-2 levels increase in children following cardiopulmonary bypass. Intensive Care Med 2008, 34:1851-1857.

12. Lovegrove FE, Tangpukdee N, Opoka RO, Lafferty El, Rajwans N, Hawkes M, Krudsood S, Looareesuwan S, John CC, Liles WC, Kain KC: Serum angiopoietin-1 and -2 levels discriminate cerebral malaria from uncomplicated malaria and predict clinical outcome in African children. Plos One 2009, 4:e4912.

13. van Meurs M, Kumpers P, Ligtenberg JJ, Meertens JH, Molema G, Zijlstra JG Bench-to-bedside review: Angiopoietin signalling in critical illness a future target? Crit Care 2009, 13:207

14. Lukasz A, Hellpap J, Horn R, Kielstein JT, David S, Haller H, Kumpers P: Circulating angiopoietin- 1 and angiopoietin-2 in critically ill patients: development and clinical application of two new immunoassays. Crit Care 2008, 12:R94.

doi:10.1186/cc9090

Cite this article as: David S, et al.: Does low angiopoietin-1 predict adverse outcome in sepsis? Critical Care 2010, 14:180. 